(Aus der Universitätsklinik für Dermatologie und Syphilidologie in Wien [Vorstand: Hofrat Prof. Dr. G. Riehl].)

\title{
Über die Berechtigung der Aufstellung von charakteristischen Kurventypen der Goldansflockung des Liquors bei luogenen Affektionen des Zentralnervensystems.
}

\author{
Von \\ L. Arzt und H. Fuhs, \\ Assistenten der Klinik. \\ Mit 1 Textabbildung, \\ (Eingegangen am 28. Juni 1921.)
}

Der hohe Wert, der in zahlreichen Arbeiten über die Goldsolreaktion dieser kolloidchemischen Methode für die Liquordiagnostik zugeschrieben wird, beruht neben ihrer größeren Feinheit und Empfindlichkeit vor allem darauf, daß wir in der Goldreaktion keine quantitative, sondern der Hauptsache mach eine qualitative Reaktion vor uns haben. Da das Ausflockungsmaximum, je nach dem Widerspiel von schützenden und hemmenden Kräften im pathologischen Liquor, bei den verschiedenen Erkrankungsformen eine differente Lage, Breite und Tiefe aufweist, sind somit nicht selten weitgehende Schlüsse auf die Art der Nervenerkrankung möglich. Doch nicht nur für ätiologisch getrennte Krankheitsbilder sind typische Füllungszacken beschrieben, sondern auch für verschiedene Erkrankungsformen des Nervensystems gleichen Ursprunges eigene Kurventypen festgelegt worden. Wir denken dabei an das den Dermatologen besonders beschäftigende Gebiet der luogenen Nervenaffektionen. Eskuchen ${ }^{1}$ ) hat dafür nach Langes ${ }^{2}$ ) Muster eine Reihe von charakteristischen Kurventypen aufgestellt, deren Specifität für die einzelnen Krankheitsbilder bereits von manchen Autoren [Weigeld $t^{3}$ ), Kyrle, Mras und Brandt ${ }^{4}$ ) u.a.] auf Grund ihrer Beobachtungen angezweifelt wurde.

Auch wir haben an dem Material der Klinik eingehende Nachforschungen über die Berechtigung der Eskuchen schen Kurventypen bei

1) Eskuchen, Die Lumbalpunktion. 1919. Urban \& Schwarzenberg.

2) Lange, Berl, klin. Wochenschr. 1912, Nr. 19.

3) Weigeldt, Dtsch. Zeitschr. f. Nervenheilk. 6r, Nr. 5/6. 1921.

*) Kyrle, Mras und Brandt, Wien. klin. Wochenschr. 1920, Nr. 1. 
Lues angestellt und wollen die Ergebnisse nach den einzelnen Flockungsformen gesondert erörtern. Anbei soll auch noch einiger von uns gefundenen atypischen Kurven bei pathologisch veränderten Luetikerliquores und solchen von der Norm abweichenden Typen, die nach allem von uns als negativ bezeichnet werden müssen, gedacht werden.

Die versehiedenen Goldsol-Kurventypen nach Eskuchen.

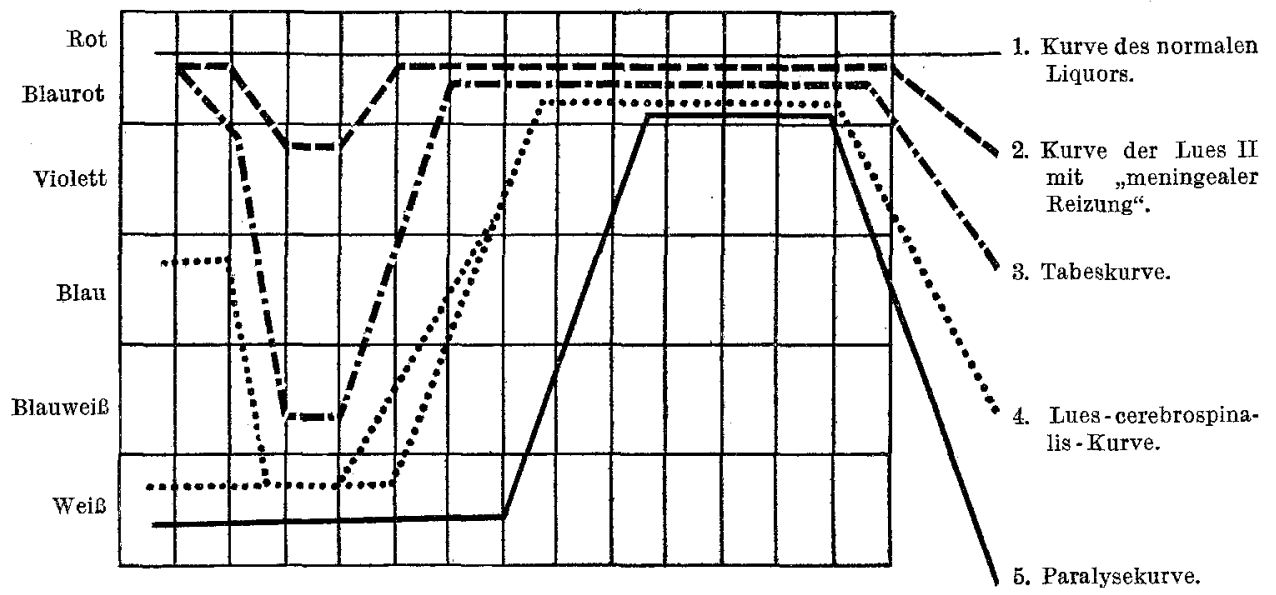

I. Paralysekurve.

Den sog. Paralysetypus (Tabelle, Kurve 5) und eine ihm zumindest sehr ähnliche Ausflockungsform fanden wir insgesamt bei 11 Liquores, und zwar bei : 1 Paralysis progr., 2 Tabes dors., I Lues cerebrospinalis, 2 Lues latens (15 Jahre und 18 Jahre seit der Infektion), 2 Rezidivexanthemen im Frühstadium der Lues mit Leukoderm, sämtliche mit in Spuren positivem Nervenbefund, endlich 1 Erstlingsexanthem mit Leukoderm, 1 Encephalitis haemorrhagica nach Salvarsan im Frühstadium der Lues und 1 Rezidivexanthem mit Periostitis luetica der Tibien, letztere 3 Patienten mit negativem klinischen Nervenbefund. Sämtliche Liquores zeigten auch in den übrigen Reaktionen positiven Befund. Somit müssen wir nach unserem Resultate vorläufig einen nur für Paralyse typischen Ausflockungsvorgang, wie ihn Eskuchen aufstellt, ablehnen, da auch bei anderen Fällen, wo nicht das kleinste Symptom auf Paralyse deutete, dieser Kurventyp zu finden war. Immerhin wäre zu erwägen, ob nicht derartige Fälle Paralysekandidaten darstellen, von der Auffassung ausgehend, daß das Schicksal quoad späterer Erkrankung des Zentralnervensystems jedes Patienten bereits bei der ersten Aussaat des Virus entschieden ist $\left[K_{y r l e}{ }^{1}\right)$ u. a.]. Es wären

1) K yrle, Über den derzeitigen Stand der Lehre von der Pathol. und Ther. der Syph. 1919. 
von charakteristischen Kurventypen der Goldausflockung des Liquors usw. 209

daher diese Patienten genauest im Auge zu behalten, ihr Liquor öfter zu kontrollieren und sie neurologischerseits durch lange Zeit zu überwachen. Für letztere Annahme möchte fast der Krankheitsverlauf eines Patienten mit neurologisch feststehender Lues cerebrospinalis sprechen, der im Liquor neben sonst positiven Reaktionen einen der Cerebrospinaliskurve ähnlichen Ausflockungstypus zeigte; der Patient gab nach einer kombinierten Quecksilbersulfoxylatkur die typische Paralysekurve. Nach einem halben Jahr erfuhren wir, daß er an beginnender Paralyse erkrankt sei. Es hat also hier noch vor neurologischer Feststellbarkeit der Paralyse die Goldreaktion das Schicksal des Patienten angekündigt.

\section{Tabeskurve.}

Tabeskurven (Tab., Kurve 3) oder ihnen ähnliche hatten wir bei 11 Liquores; sie betrafen 3 Tabes dorsalis, 2 Lues cerebr., 2 Lues latens mit auch sonst positivem Liquorbefund, 3 Rezidivexantheme mit gleichfalls in den übrigen Peaktionen positiver Cerebrospinalflüssigkeit und negativem, klinischen Nervenbefund in zwei, geringem Nervenbefund in einem Fall, endlich als Rarität eine seronegative Sklerose bei negativem Nervenbefund und sonst nur geringfügiger Globulinvermehrung. Es kann also auch von einer typischen Tabeskurve nach unseren Erhebungen nicht gesprochen werden, indem unter 11 derartigen Kurventypen nur 3 das spezielle Krankheitsbild betrafen und auch hier nicht ohne weiteres angenommen werden kann, dab aus all den anderen Fällen sich späterhin noch Tabes entwickeln werde. Fine unter unseren Tabeskurven bei einem Rezidivexanthem und auch sonst komplett positivem Liquor zeigte eine günstige Beeinflussung durch kombinierte Hg-Salvarsantherapie. Es spricht dies auch gegen die Ankündigung einer etwa in der Entwicklung begriffenen Tabes durch die Goldreaktion, da in einem derartigen Falle von zu Recht bestehenderTabes nach unserer Beobachtung die Kur fast niemals eine auch nur leichte Besserung der Ausflockung, meist nur ein Stationärbleiben oder Verschlechterung der Goldsolkurve nach sich zieht.

\section{Kurve der Lues cerebrospinalis.}

(Kombination von Tabes- und Paralysetypus.)

Die Lues-cerebrospinalis-Kurve ('Tab., Kurve 4) oder eine ihr annähernd gleichende zeigten 6 Patienten im Liquor, darunter 2 neurologisch feststehende Formen von Lues cerebrospinalis, 1 Tabes dors., 2 ältere Stadien latenter Lues mit fast komplett positivem Liquor, der auf eine kombinierte Kur zwar einen geringen Rückgang der Globulinwerte, dafür aber eine Verschlechterung der Goldsolreaktion aufwies. Also auch bei dieser dritten Form der luogenen Nervenerkrankung ließ uns dieser Kurventypus teilweise im Stich. 
IV. Kurve der Lues II mit „,meningealer Reizung“"

Einen weiteren Kurventypus Eskuchens stellt die „,meningeale Reizungskurve" dar (Tabelle, Kurve 2). Sie wurde von Eskuchen so bezeichnet, da sie bei Personen mit positiver WaR. im Blute, aber ohne klinische Zeichen von seiten des Zentralnervensystems (Lues latens) vorkommt und nach ihm als letzter Überrest einer im sekundären Stadium durchgemachten Meningitis (meningitische Reizung) zu deuten ist. Doch wird sie von ihm schon als diagnostisch nicht ganz verwertbar erachtet, indem sie ganz selten auch bei sicher nichtluetischen Personen vorkommen soll. Wir fanden diese Kurve unter 375 Fällen annähernd bei 24 Liquores (ca. $7 \%$ ) und da bei nahezu allen Stadien der Lues, bei seronegativer und seropositiver Primärlues, Erstlings- und Rezidivexanthemen, Gummen, Mesaortitis Iuetica und Lues latens und auch bei einem Fall von Lupus erythematosus und nichtspezifischer Alopecie ohne Lues. Bei nahezu sämtlichen Liquores waren die übrigen Reaktionen und Nervenbefunde negativ. Ausnahmen bildeten ein krustöses Syphilid, bei dem erhöhte Zellzahl und Eiweißgehalt bestand, sowie eine seropositive Primärlues und ein Erstlingsexanthem mit in Spuren vermehrtem Globulingehalt. Es besteht somit auch hier, wie ja schon Eskuchen zum Teil zugibt, der Kurventypus nicht zu Recht.

\section{Kurve des normalen Liquors.}

Die Kurve des normalen Liquors (Tabelle, Kurve 1), id est fehlende Ausflockung und Erhaltenbleiben der normalen Goldsolfarben in allen Röhrchen weisen 38 Liquores aus verschiedenen Stadien der Lues, der Hauptsache nach jedoch der Frühperiode, bei sonst negativem Ausfall der übrigen angestellten Reaktionen auf. Eine Ausnahme bilden 5 Liquores bei Lues II mit erhöhter Zellzahl, einer mit positiver GuillainParantscher Reaktion und zwei mit in Spuren positiver Pandyreaktion als einziger sonst vorhandener Reaktion, die wohl auch nur als Zeichen einer allgemeinen, luetischen Infektion hier in Betracht kommen dürften.

\section{VI. „Aty pische Goldsolkurven."}

Noch eine weitere, von Eskuchen nicht geführte Gruppe von Kurven soll Erwähnung finden, die man evtl. als , at y pische Gold solkurven" bezeichnen könnte. Wir hatten nämlich auch bei 97 Fällen leichte Ausflockungen bis höchstens blaurot, in den Verdünnungen zwischen $1: 10$ und $i: 160$ die verschiedensten Zacken ergebend, zu verzeichnen, die wir nach Erwägung aller Krankheitssymptome noch als negativ zu werten uns veranlaßt sahen, Diese Verschiedenheit dürfte auf den schon in früheren Arbeiten von uns erwähnten differenten Dispersitätsgrad und somit ungleiche Empfindlichkeit der Goldlösungen zurückzuführen sein. Wir fanden in 44 dieser Fälle, 
also nahezu der Hälfte, geringfügige positive Zell- und Globulinwerte bei sonst negativer NissI- und Wassermannreaktion sowie negativem Nervenbefund; nur in 2 Fällen war letzterer in Spuren positiv. - Wir konnten somit auch da den erhöhten Zell- und Eiweißwerten nur eine bedingte, nämlich allgemeine Abwehrrolle zuerkennen.

Ein zweiter Teil von derartig atypischen Kurven entfällt auf Fälle mit deutlich pathologisch verändertem Liquor und zeigt auch dementsprechend tiefere Lage des Ausflockungsmaximums : dieses wird zwar in den für Lues charakteristischen Verdünnungen $(1 / 40-1 / 80$ ) regelmäßig eingehalten, doch treten außerdem noch andere Momente hinzu, die der Kurve ein ganz verschiedenes, oft bizarres Aussehen verleihen, ohne daß wir imstande sind, nähere Beziehungen zu der jeweils zugehörigen Luesform zu ziehen. Verbreiterung der Kurve am tiefsten Punkt, Verschiebungen der Ausflockung bald nach links, bald nach rechts, staffelförmiges Ansteigen der Kurve von der stärksten gegen die schwächeren Liquorkonzentrationen, zweiZacken, eine typische und atypische, treten in bunter Reihenfolge hervor. Dabei muß betont werden, daß bei allen diesen Kurventypen Blutbeimengungen im mikroskopischen Bilde oder Fehler in der Technik der Reaktion durch mehrfache Kontrollen mit verschiedenen Lösungen ausgeschlossen werden konnten.

Nach dem Ergebnis unserer Untersuchungen kann der Aufstellung typischer Kurven für die vereinzelten Formen luogener Nervenerkrankungen nicht allgemein beigestimmt werden. Wir verwerten die Goldsolreaktion zur Diagnose luogener Nervenerkrankungen, sind jedoch derzeit nicht in der Lage, aus der Form der Kurve einen sicheren Schluß auf die Art der luogenen Nervenerkrankung zu ziehen. 